\title{
Bestimmung der Atemstromstärke mithilfe des ThorAKUSTIK-Verfahrens bei Mund- und Nasenatmung
}

\author{
ThorAKUSTIK - A Computer-Based Method to Determine the Oronasal \\ Respiratory Flow
}

Autoren

Institute

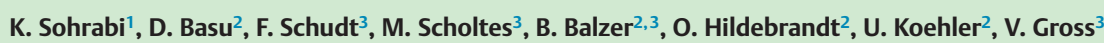

${ }^{1}$ ThoraTech GmbH, Anwenderzentrum Medizintechnik, Gießen

2 Klinik für Innere Medizin, SP Pneumologie, Intensiv- und Schlafmedizin, Philipps-Universität Marburg

3 Biomedizinische Technik, Fachbereich KMUB, Technische Hochschule Mittelhessen, Gießen eingereicht 13.7.2012 akzeptiert nach Revision 28. 8. 2012

\section{Bibliografie}

Dol http://dx.doi.org/ 10.1055/s-0032-1325692

Online-Publikation: 26.9.2012

Pneumologie 2012; 66: 669-673

(c) Georg Thieme Verlag KG

Stuttgart · New York

ISSN 0934-8387

Korrespondenzadresse

Prof. Dr. Volker Groß

Biomedizinische Technik,

Fachbereich KMUB

Technische Hochschule

Mittelhessen

Wiesenstraße 14

35390 Gießen

volker.gross@kmub.thm.de

\section{Zusammenfassung \\ V}

Hintergrund: Die Atemflussbestimmung stellt ein wichtiges Verfahren zur Detektion von schlafbezogenen Atmungsstörungen dar. In der Polysomnografie können unter Verwendung der Standardmethoden allerdings nur qualitative Aussagen über den Atemfluss getroffen werden. Quantitative Methoden wie die Pneumotachografie sind für Messungen während des Schlafes wegen der Größe der Sensoren ungeeignet.

Methode: Vor diesem Hintergrund wurde das ThorAKUSTIK-Verfahren zur akustischen Atemflussbestimmung entwickelt. Mithilfe dieses Verfahrens wurde anhand des trachealen Atemgeräusches die Atemstromstärke bestimmt. Zur Evaluation des neuen Verfahrens wurde eine Vergleichsmessung mit dem Pneumotachografen durchgeführt und anschließend die Korrelationskoeffizienten berechnet.

Patienten: In der vorliegenden Studie wurde ein homogenes Kollektiv aus 20 männlichen, nichtrauchenden Probanden ausgewählt.

Ergebnisse: Es konnte gezeigt werden, dass das ThorAKUSTIK-Verfahren zuverlässig die Atemflussstärke bestimmt und kontinuierlich darstellen kann. Es fanden sich sehr signifikante Korrelationen $(r=0,89$ bis 0,$91 ; p<0,01)$ zwischen dem tatsächlichen Atemfluss und dem mittels ThorAKUSTIK berechneten Atemfluss.

Schlussfolgerungen: Das neue ThorAKUSTIK-Verfahren erlaubt sowohl ein Langzeitmonitoring als auch eine Live-Überwachung des Atemflusses und kann dadurch zukünftig sehr variabel und in verschiedenen klinischen Bereichen eingesetzt werden. Hierzu sind aber zunächst noch weitere Studien mit einem größeren Kollektiv notwendig, um die jeweilige Anwendung im klinischen Alltag zu verifizieren.

\section{Abstract \\ $\nabla$}

Background: Respiratory flow detection with the aim of detecting sleep-related breathing disorders plays a major role in polysomnography. Due to the fact that pneumotachographs are too bulky and not suitable for measurements during sleep, the ThorAKUSTIK system has been developed. By attaching a noise sensor right next to larynx, it determines the respiratory flow in an acoustic way.

Methods: The ThorAKUSTIK system as well as a pneumotachograph were applied simultaneously. The correlation between those two methods has been calculated.

Patients: We investigated twenty male subjects. All of them were non-smokers.

Results: The ThorAKUSTIK-System showed a highly positive correlation ( $\mathrm{r}=0.89$ to $0.91 ; \mathrm{p}$ $<0.01$ ) and was able to measure the respiratory flow in a reliable way.

Conclusions: The ThorAKUSTIK-System allows a long-term live monitoring and has the potential to be used in several clinical departments. Larger studies are necessary to verify the application in the clinical routine. 


\section{Hintergrund \\ $\nabla$}

Die Atemflussbestimmung stellt ein wichtiges Verfahren zur Detektion von schlafbezogenen Atmungsstörungen dar. Hierzu wird häufig ein sogenannter Thermistor verwendet, der den Temperaturunterschied zwischen ein- und ausgeatmeter Luft registriert. Dieses Verfahren lässt allerdings lediglich qualitative Aussagen über den Atemfluss zu [1]. Ein präziseres Verfahren ist die orale Atemflussmessung mittels Pneumotachograf. Vorteil hierbei ist, dass das tatsächlich fließende Volumen pro Zeiteinheit gemessen werden kann. Der Nachteil des Pneumotachografen liegt darin, dass er zwar zusammen mit einer Schlafmaske verwendet werden kann, diese jedoch den Schlafkomfort des Patienten stark einschränkt. Zudem kann durch eventuell auftretende Leckagen das Messergebnis verfälscht werden.

Eine für den Patienten kaum störende Möglichkeit zur Atemflussmessung besteht in der akustischen Bewertung der Atemgeräusche über der Trachea. So fand Forgacs schon 1970 bei kleinen Atemstromstärken einen linearen Zusammenhang zwischen der Geräuschintensität und dem Atemfluss [2], welcher bei größeren Atemstromstärken exponentiell wird. Untersuchungen von Soufflet zeigten, dass es einen Schwellenwert gibt, ab dem die Detektion des Atemflusses überhaupt erst möglich ist. Dieser lag im Allgemeinen bei 0,1 bis $0,2 \mathrm{l} / \mathrm{s}$. Außerdem zeigte sich eine hohe Variabilität zwischen den einzelnen Probanden. Bei der Bestimmung der trachealen Flussraten ergaben sich mittlere Fehler von 13-15\% [3]. Mussell führte unterschiedliche Berechnungen für die Inspiration und Exspiration ein [4].

Die trachealen Atemgeräusche, die in den oberen Atemwegen im Bereich von Pharynx und Larynx entstehen, lassen sich auf turbulente Luftströmungen zurückführen, welche die Trachealwand zum Vibrieren bringen [5]. Die trachealen Atemgeräusche haben ein breites Frequenzspektrum mit einem relativ gleichbleibenden Frequenzanteil zwischen 75 und $1000 \mathrm{~Hz}$ [6]. Oberhalb dieser Grenzfrequenz fällt das Spektrum steil ab. Vereinzelt werden jedoch Frequenzanteile bis $1600 \mathrm{~Hz}$ beschrieben. Lessard untersuchte 1986 den Zusammenhang zwischen dem Frequenzspektrum und der Atemflussrate. Auch hier wurde ein linearer $\mathrm{Zu}-$ sammenhang zwischen der mittleren Frequenz des trachealen Atemgeräusches und dem Fluss nachgewiesen, wobei ab Werten von $0,75 \mathrm{l} / \mathrm{s}$ die mittlere Frequenz gleich blieb. Außerdem stellte Lessard fest, dass die mittleren Frequenzen der Exspiration höher sind, als die der Inspiration [7]. Moussavi fand 2002 einen linearen Zusammenhang zwischen der mittleren Bandpower (Frequenzstärke) im Bereich von 100 bis $600 \mathrm{~Hz}$ und dem Atemfluss [8].

Vor diesem Hintergrund entwickelte die ThoraTech GmbH das ThorAKUSTIK Verfahren, welches in Kombination mit einem neuen Geräuschsensor die automatische Detektion und Bewertung des trachealen Atemflusses ermöglicht. Dabei wird der Sensor, ähnlich einer EKG-Elektrode, neben dem Kehlkopf angebracht und die entstehenden Atemgeräusche werden aufgezeichnet. Mit diesem Verfahren können sowohl die nasale als auch die orale Atmung detektiert werden. Der Auskultationsort für das tracheale Atemgeräusch ist leicht zugänglich und eignet sich auch im Rahmen der Schlafdiagnostik für die Analyse nächtlicher Apnoephasen [9]. Ein quantitatives und gleichzeitig für den Patienten komfortables Verfahren, wie es mit ThorAKUSTIK erreicht werden könnte, ist vor allem im Langzeit-Monitoring und in der Polysomnografie wünschenswert.
Tab. 1 Einschlusskriterien.

\begin{tabular}{ll}
\hline Parameter & Wert \\
\hline Geschlecht & männlich \\
\hline Alter & $20-40$ Jahre \\
\hline Lungenfunktionstest & $\mathrm{FEV}_{1}>80 \%$ \\
\hline letzte Infektion & $>2$ Monate \\
\hline Nichtraucher & seit $>5$ Jahren \\
\hline maximale Packyears & $5 \mathrm{PY}$ \\
\hline BMI & $17-30 \mathrm{~kg} / \mathrm{m}^{2}$ \\
\hline
\end{tabular}

\section{Studienziel \\ $\nabla$}

Im Rahmen der vorliegenden Studie sollte das neue ThorAKUSTIK-Verfahren mit einem in einer Fullface-Maske integrierten Pneumotachografen als Referenzmethode verglichen werden. Die primäre Hypothese lautete, dass die beiden Verfahren zur Atemflussbestimmung qualitativ und quantitativ gleichwertig sind. Dies soll sowohl für die Inspiration als auch für die Exspiration und jeweils sowohl für Mund- als auch Nasenatmung gelten. Um eine Vergleichbarkeit zu gewährleisten, sollte mit beiden Verfahren gleichzeitig der Atemfluss kontinuierlich bestimmt und anschließend statistisch bewertet werden.

\section{Probanden und Methoden \\ $\nabla$}

Probanden

Für die Studie wurde ein homogenes Kollektiv aus 20 männlichen, nichtrauchenden Probanden rekrutiert. Die Einschlusskriterien für das Probandenkollektiv sind in Tab. 1 dargestellt. Alle Untersuchungen wurden in der Klinik für Innere Medizin, Schwerpunkt Pneumologie des Universitätsklinikums Gießen und Marburg GmbH, Standort Marburg, durchgeführt. Vor Beginn der Messungen wurden alle Probanden mündlich und schriftlich umfassend über Inhalt und Ablauf der Studie informiert, und jeder Proband unterzeichnete eine Einwilligungserklärung. Das Studienvorhaben wurde zuvor durch die Ethikkommission des Fachbereichs Medizin der Universität Marburg geprüft und genehmigt.

\section{Materialien}

Die Lungenfunktionsmessung zur Beurteilung der Lungengesundheit wurde mit dem Bodyplethysmografen Master Screen Body (Jäger, Würzburg) und dem Spirometer Master-Screen CPX (Jäger, Würzburg) durchgeführt. Die Aufzeichnung und automatische Online-Analyse der Atemgeräusche erfolgte mithilfe des ThorAKUSTIK-Systems (ThoraTech GmbH, Gießen). Die damit durchgeführte computergestützte, elektronische Auskultation orientiert sich an den Empfehlungen des Computerized-Respiratory-Sound-Analysis-Standards (CORSA) [10]. Die Messungen erfolgten nach einer vorherigen patientenindividuellen Kalibrierung. Die automatische Berechnung basiert auf einer FFT-Spektralanalyse des trachealen Atemgeräuschs (siehe Abb.1). Da bei sehr niedrigen Flusswerten aufgrund der ausschließlich laminaren Strömung keine Atemgeräusche entstehen können, werden Werte unterhalb eines Schwellenwertes von 0,2 1/s nicht berechnet.

Für die Referenzmessung wurde ein Pneumotachograf (Handpneumotachograf, Jäger, Würzburg) mit Fullface-Maske (NV full face Maske (ohne Auslassöffnungen) RESMED Inc., San Diego, 


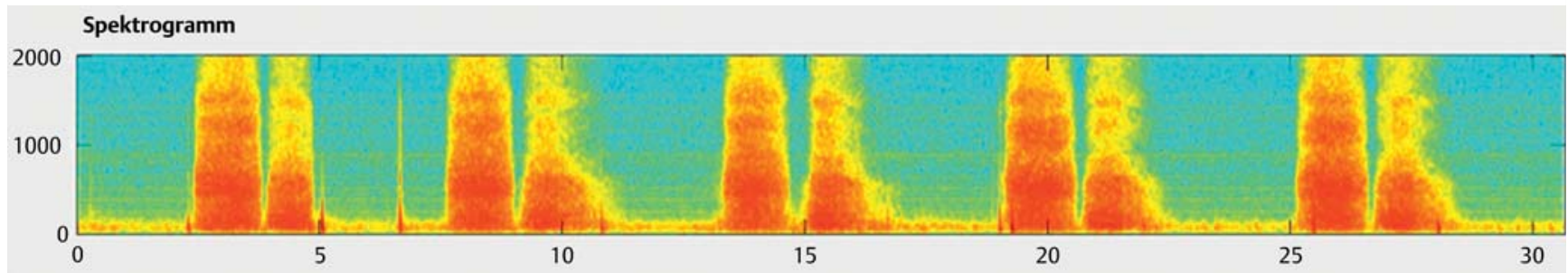

Abb. 1 FFT-Spektralanalyse des trachealen Atemgeräusches für ThorAKUSTIK-online-Berechnung.

Tab. 2 Ergebnisse der Lungenfunktionsmessungen.

\begin{tabular}{|c|c|c|c|c|c|c|c|c|c|c|}
\hline \multirow[t]{2}{*}{ Nr. } & \multirow[t]{2}{*}{ Alter (J) } & \multirow[t]{2}{*}{ BMI $\left(\mathbf{k g} / \mathbf{m}^{2}\right)$} & \multirow[t]{2}{*}{ FEV 1 (L) } & \multirow{2}{*}{$\begin{array}{l}\text { FEV1 } \\
\text { (\% Soll) }\end{array}$} & \multirow[t]{2}{*}{ FVC (L) } & \multirow{2}{*}{$\begin{array}{l}\text { FVC } \\
\text { (\% Soll) }\end{array}$} & \multicolumn{2}{|c|}{ Fullface $r$} & \multicolumn{2}{|c|}{ Nasenmaske $r$} \\
\hline & & & & & & & Insp. & Exsp. & Insp. & Exsp. \\
\hline 1 & 25 & 24,49 & 5,01 & 107,5 & 5,67 & 102,2 & 0,96 & 0,96 & 0,82 & 0,95 \\
\hline 2 & 22 & 22,59 & 5 & 103,6 & 6,53 & 112,9 & 0,96 & 0,96 & 0,94 & 0,94 \\
\hline 3 & 25 & 24,02 & 4,82 & 99,9 & 5,38 & 93 & 0,90 & 0,93 & 0,98 & 0,95 \\
\hline 4 & 24 & 21,74 & 3,75 & 81,3 & 5,04 & 91,8 & 0,80 & 0,90 & 0,91 & 0,79 \\
\hline 5 & 24 & 23,30 & 4,36 & 99,1 & 5,09 & 97,7 & 0,93 & 0,93 & 0,96 & 0,95 \\
\hline 6 & 24 & 26,60 & 5,4 & 110,9 & 6,37 & 109,1 & 0,84 & 0,94 & 0,73 & 0,78 \\
\hline 7 & 20 & 18,31 & 4,92 & 107,7 & 5,18 & 95,2 & 0,98 & 0,95 & 0,89 & 0,90 \\
\hline 8 & 26 & 22,10 & 5,23 & 113,1 & 6,42 & 116,2 & 0,60 & 0,71 & 0,81 & 0,84 \\
\hline 9 & 26 & 22,45 & 4,19 & 89,7 & 4,84 & 86,7 & 0,86 & 0,87 & 0,90 & 0,92 \\
\hline 10 & 25 & 21,84 & 5,27 & 107,4 & 6,83 & 115,8 & 0,91 & 0,90 & 0,88 & 0,84 \\
\hline 11 & 20 & 19,72 & 4,34 & 83,9 & 5,7 & 91,3 & 0,70 & 0,84 & 0,97 & 0,91 \\
\hline 12 & 25 & 24,62 & 4,5 & 92,3 & 5,83 & 99,9 & 0,78 & 0,88 & 0,78 & 0,78 \\
\hline 13 & 25 & 20,99 & 4,06 & 89,8 & 4,41 & 82,1 & 0,92 & 0,92 & 0,84 & 0,90 \\
\hline 14 & 28 & 25,50 & 4,66 & 109,3 & 5,57 & 109,9 & 0,89 & 0,86 & 0,89 & 0,84 \\
\hline 15 & 26 & 25,34 & 4,35 & 95,9 & 5,2 & 96,1 & 0,95 & 0,91 & 0,89 & 0,79 \\
\hline 16 & 36 & 19,66 & 4,3 & 104,8 & 5,63 & 113 & 0,93 & 0,94 & 0,95 & 0,85 \\
\hline 17 & 27 & 25,00 & 5,37 & 101,7 & 6,83 & 106,4 & 0,95 & 0,96 & 0,90 & 0,96 \\
\hline 18 & 25 & 20,99 & 5,74 & 112 & 5,98 & 96,7 & 0,83 & 0,78 & 0,83 & 0,79 \\
\hline 19 & 25 & 20,59 & 5,14 & 106,6 & 6,09 & 105,4 & 0,88 & 0,96 & 0,92 & 0,83 \\
\hline 20 & 24 & 24,93 & 3,79 & 85,3 & 5,83 & 110,9 & 0,96 & 0,96 & 0,95 & 0,96 \\
\hline MW & 25.1 & 22.74 & 4.71 & 100.09 & 5.72 & 101.62 & 0,90 & 0,92 & 0,91 & 0,89 \\
\hline Stw & 3.24 & 2.28 & 0.56 & 9.96 & 0.66 & 10.04 & 0,10 & 0,07 & 0,07 & 0,07 \\
\hline
\end{tabular}

USA) verwendet. Das gemessene Pneumotachograf-Signal und das berechnete ThorAKUSTIK-Signal wurden zeitgleich aufgezeichnet und anschließend analysiert.

\section{Versuchsablauf}

Nach dem Aufklärungsgespräch wurde zu Beginn jeder Untersuchung eine ausführliche Anamnese mit Fokus auf eventuell vorliegende Erkrankungen des Respirationstraktes durchgeführt. Es folgte eine ausführliche körperliche Untersuchung. Anschließend wurde der Proband zur Verifizierung der Lungengesundheit einem Lungenfunktionstest zur Messung der Zielgrößen $\mathrm{FEV}_{1}$ und FVC unterzogen. Die Werte der BMI- und Lungenfunktionsmessung sind in Tab. 2 aufgeführt. Abschließend wurde, wie folgt, die ThorAKUSTIK-Messung durchgeführt: Nach einer individuellen Kalibrierung atmete der Proband jeweils 4 Minuten lang durch den Mund (bei verschlossener Nase) in eine Fullface-Maske und anschließend durch eine Nasenmaske (bei geschlossenem Mund), an die jeweils ein Pneumotachograf angeschlossen war (siehe Abb.2). Die Reihenfolge sowie der zeitliche Verlauf der Untersuchung ist in Abb. 3 dargestellt. Die Aufnahmen wurden in 30-Sekunden-Blöcken abgespeichert, sodass sich bei einem 4 Minuten andauernden Zyklus insgesamt 16 Aufzeichnungsblöcke pro untersuchtem Probanden ergaben.

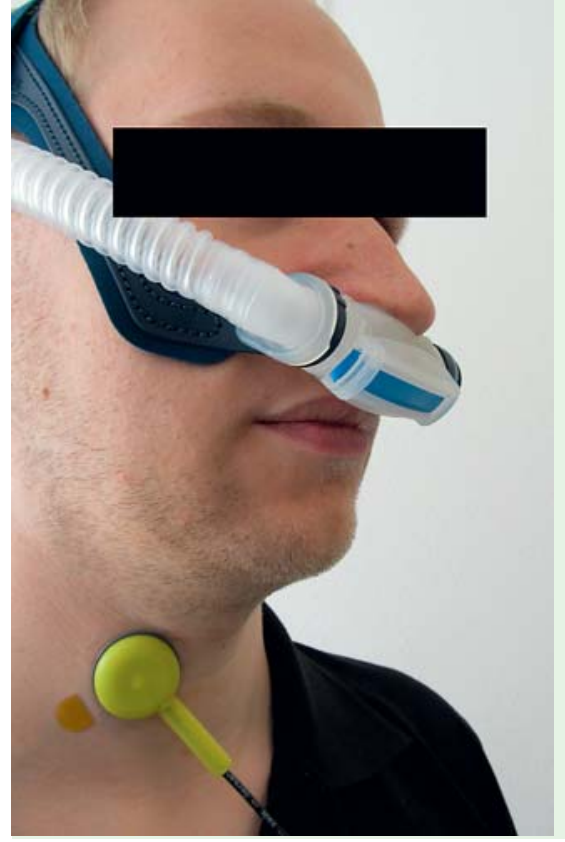

Abb.2 Schematischer Messaufbau. 


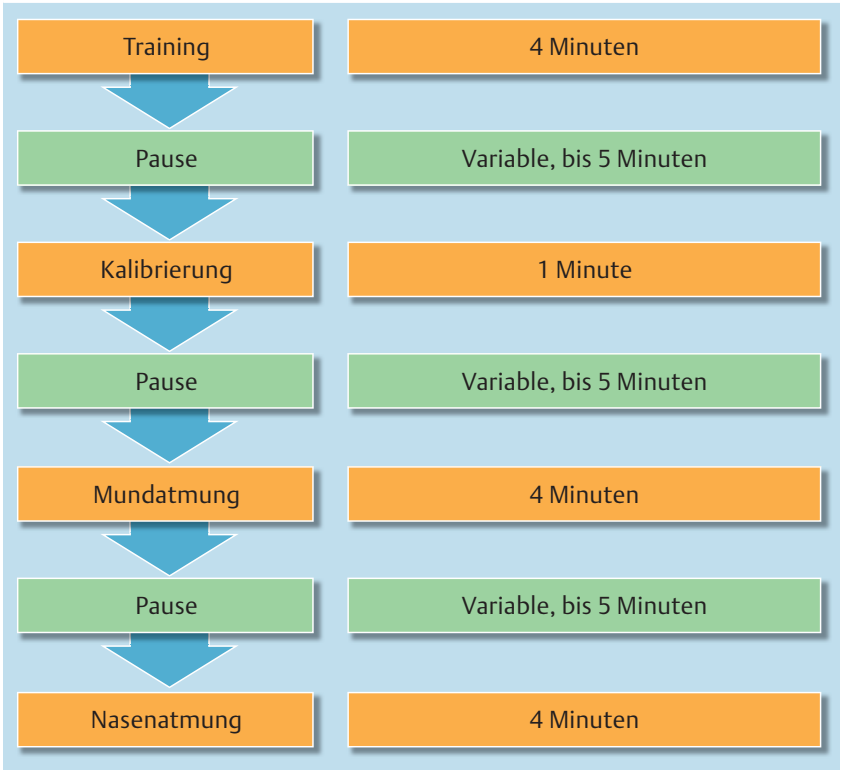

Abb.3 Zeitlicher Messverlauf.

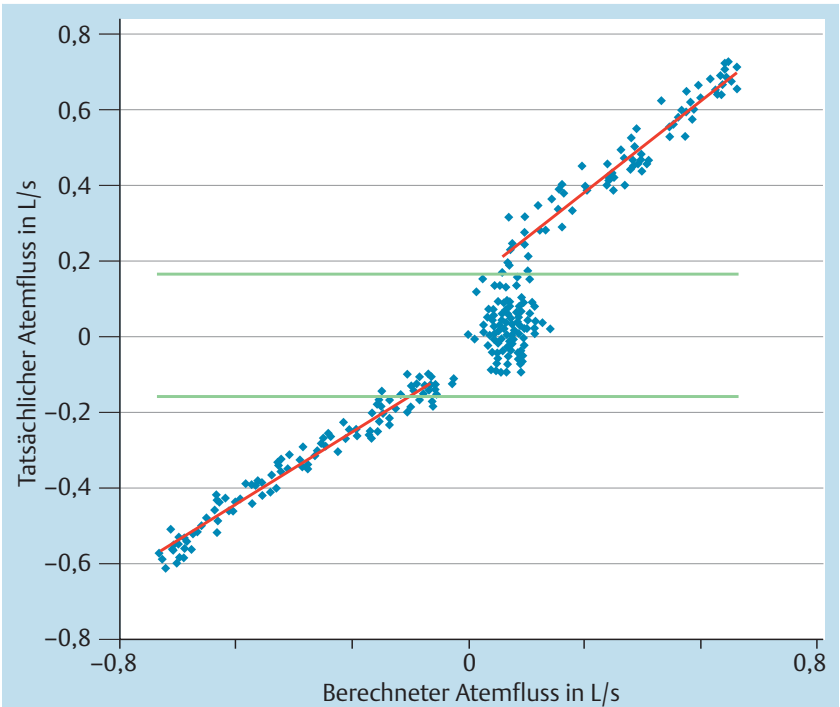

Abb.4 Exemplarisches Streudiagramm mit Regressionsrechnung für Inspirations- und Exspirationsphase.

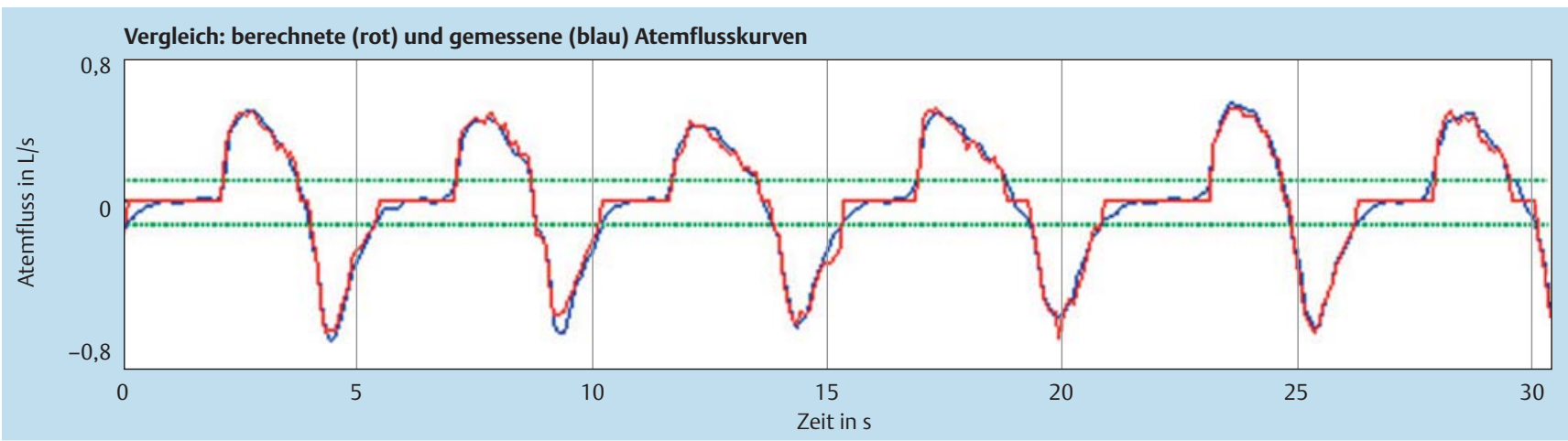

Abb.5 Gegenüberstellung der tatsächlichen (blau) und der berechneten Atemflusskurve (rot).

\section{Statistik}

Die statistische Übereinstimmung der Atemflusskurven beider Messsysteme wird durch den Korrelationskoeffizienten ( $r$ ) nach Pearson dargestellt. Er wurde für jeden Probanden jeweils für die Inspirations- und Exspirationsphase sowohl bei Nasen- als auch bei Mundatmung berechnet. Das Signifikanzniveau wurde auf $\mathrm{p}=0,01$ festgelegt. Die statistischen Berechnungen wurden mit der MATLAB-Statistics Toolbox (Mathworks Inc., Natick, Massachusetts, USA) durchgeführt.

\section{Ergebnisse}

Im Rahmen der individuellen Kalibrierung wurden die Regressionsgeraden für Mund- und Nasenatmung jeweils für Inspiration und Exspiration getrennt ermittelt. Ein Beispiel für eine Gegenüberstellung der beiden Werte befindet sich in $\bullet$ Abb.4. Anschließend wurden beide Werte online am Bildschirm dargestellt und zeitgleich aufgezeichnet (siehe Abb.5).

Für die statistische Auswertung wurden die Korrelationskoeffizienten separat für nasale und orale Messung berechnet. Außerdem wurde jeweils zwischen Inspirations- und Exspirationsphase unterschieden. Für die Bestimmung der mittleren Koeffizien-
Tab. 3 Mittlere Korrelationswerte (MW) und die Standardabweichungen (Stw.) zwischen den berechneten und gemessenen Flowwerten, getrennt für Nasen- und Mundatmung sowie für Inspirations- und Exspirationsphase.

\begin{tabular}{llll} 
& & Inspiration & Exspiration \\
\multirow{2}{*}{ Mundatmung } & MW (r) & $0,90^{* *}$ & $0,92^{* *}$ \\
\cline { 2 - 4 } Nasenatmung & Stw. $(r)$ & 0,10 & 0,07 \\
\cline { 2 - 3 } & MW (r) & $0,91^{* *}$ & $0,89^{* *}$ \\
\cline { 2 - 3 } & Stw. $(r)$ & 0,07 & 0,07
\end{tabular}

Die $\mathrm{p}-$ Werte sind wie folgt codiert: ${ }^{*} \mathrm{p}<0.05$ (signifikant), ${ }^{* *} \mathrm{p}<0.01$ (sehr signifikant), ${ }^{* * *} \mathrm{p}<0.001$ (hoch signifikant).

ten wurden alle Werte Z-transformiert, über alle Probanden gemittelt und anschließend rücktransformiert. Die entsprechenden mittleren Korrelationskoeffizienten sind $\bullet$ Tab. 3 zu entnehmen. Für alle Modalitäten fanden sich sowohl für die Inspiration als auch für die Exspiration im Mittel Korrelationskoeffizienten zwischen 0,86 und 0,92. Mit p-Werten stets $<0,01$ ist die gefundene Korrelation als sehr signifikant zu bewerten. 


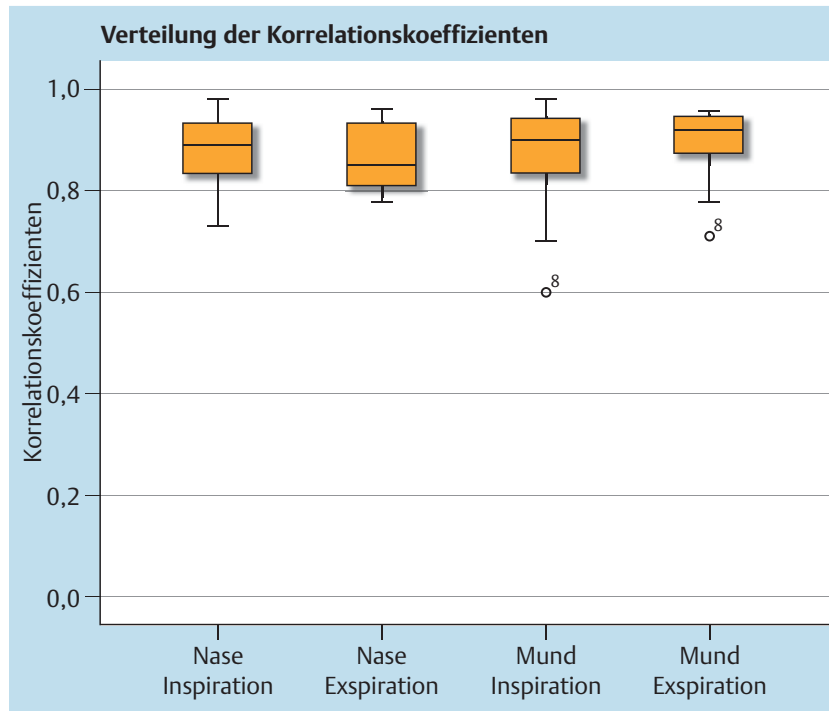

Abb.6 Boxplot zur Darstellung der Korrelationskoeffizienten, getrennt betrachtet für Mund- und Nasenatmung jeweils für die Inspirations- und Exspirationsphase.

\section{Schlussfolgerungen}

\section{$\nabla$}

In der vorliegenden Studie konnte gezeigt werden, dass die Atemflussstärke anhand des ThorAKUSTIK-Verfahrens detektiert und kontinuierlich über den Zeitverlauf dargestellt werden kann. Sowohl für Nasen- als auch für Mundatmung konnte eine sehr signifikante Korrelation zwischen dem tatsächlichen Atemfluss und dem berechneten Atemfluss gezeigt werden. Zwischen Inspiration und Exspiration ergaben sich bei den Korrelationswerten keine nennenswerten Unterschiede (siehe $\bullet$ Abb.6).

Die Ergebnisse dieser Arbeit bestätigen die Vorarbeiten von Forgacs, Soufflet, Lessard, Moussavi und Mussell und zeigen, dass mithilfe einer individuellen Kalibrierung eine Quantifizierung der Flowwerte möglich ist.

Für die praktische Anwendung im Bereich der Überwachung folgt daraus, dass zukünftig auf sehr einfache Weise der Atemfluss über längere Zeit sowohl aufgezeichnet als auch live beobachtet werden könnte (kontinuierliches Langzeit-Monitoring).
Einschränkungen des Verfahrens könnten bei plötzlichen starken Atemwegsobstruktionen wie zum Beispiel dem Beginn einer Apnoephase auftreten, da durch die starken Verengungen der Anteil turbulenter Strömungen stark ansteigt und möglicherweise als erhöhter Fluss fehlinterpretiert werden könnte.

Da diese Pilotstudie an einem ausgewählten Kollektiv unter Laborbedingungen durchgeführt wurde, liefert sie noch keine allgemein repräsentativen Ergebnisse. Daher ist geplant, in weiteren klinischen Studien das Verfahren an einem größeren Patientenkollektiv unter unterschiedlichen klinischen Bedingungen zu evaluieren und anschließend in den Markt zu überführen.

\section{Interessenkonflikt}

\section{$\nabla$}

K. Sohrabi ist Geschäftsführer der ThoraTech GmbH.

D. Basu, F. Schudt, M. Scholtes, B. Balzer, O. Hildebrandt, U. Koehler und V. Gross geben an, dass kein Interessenkonflikt besteht.

\section{Literatur}

1 Hader C, Sanner B, Rasche K. Das obstruktive Schlafapnoe-Syndrom Diagnostik. Dtsch Med Wochenschr 2004; 129: 566 -569

2 Forgacs $P$. The functional basis of pulmonary sounds. Chest 1978; 73 : $399-405$

3 Soufflet G, Charbonneau G, Polit M et al. Interaction between tracheal sound and flow rate: a comparison of some different flow evaluations from lung sounds. IEEE Trans Biomed Eng 1990; 37: 384-391

4 Mussell MJ. The need for standards in recording and analysing respiratory sounds. Med Biol Eng Comput 1992; 30: 129-139

5 Beck R, Rosenhouse G, Mahagnah M et al. Measurements and theory of normal tracheal breath sounds. Ann Biomed Eng 2005; 33: $1344-$ 1351

6 Gavriely N, Palti Y, Alroy G. Spectral characteristics of normal breath sounds. J Appl Physiol 1981; 50: 307-314

7 Lessard CS, Wong WC. Correlation of constant flow rate with frequency spectrum of respiratory sounds when measured at the trachea. IEEE Trans Biomed Eng 1986; 33: 461 - 463

8 Moussavi Z, Yap YL. Acoustic Airflow Estimation from Tracheal Sound Power. IEEE Canadian Conference on Electrical \& Computing Engineering 2002

9 Gavriely N, Cugell D. Breath Sounds Methodology. CRC Press; 1995

10 Sovijärvi ARA, Vanderschoot J, Earis JE. Computerized Respiratory Sound Analysis (CORSA): recommended standards for terms and techniques. ERS Task Force Report. European Respiratory Review 2000: 10 